Proceedings of the 47th International School and Conference on the Physics of Semiconductors "Jaszowiec 2018"

\title{
Crystallization in Topological Flat Bands in Thin Torus Limit
}

\author{
M. Kupczyński*, B. Jaworowski, P. Kaczmarkiewicz, P. Potasz and A. Wójs \\ Department of Theoretical Physics, Faculty of Fundamental Problems of Technology, \\ Wrocław University of Science and Technology, Wybrzeże Wyspiańskiego 27, PL-50370 Wrocław, Poland
}

We study a crystallization on partially filled topological flat bands of honeycomb lattice in the thin torus limit. We identify the crystallization by looking at the pair correlation densities and determine the strength of the crystallization from their Fourier transform. We observed that the crystallization depends on the parity of the denominator of the filling factor. The crystallization is weakly affected by finite size effects and its strength increases with decrease of a particle density.

DOI: 10.12693/APhysPolA.134.934

PACS/topics: 73.22.Dj, 74.20.Pq

\section{Introduction}

In recent years, the possibility of realization of quantum Hall effect on a lattice without external magnetic field has been intensely studied. Systems which exhibits non-zero Hall conductance and preserving lattice translational symmetry are known as the Chern insulators, the idea introduced by Haldane [1]. The Chern insulators are characterized by a nonzero value of an integer topological invariant named the Chern number [2], which quantized the Hall conductivity. A proposition of realization of the Chern insulators on cold atom systems $[3,4]$ was recently confirmed experimentally [5-8]. Nearly flat bands with nonzero Chern number can host the correlated phases named fractional Chern insulators (FCI) and were extensively studied numerically [9-19]. These are lattice analogues to fractional quantum Hall effect (FQHE) states and the adiabatic continuity between FCI and FQHE was shown [20, 21]. FQHE [22, 23] and FCI [24-27] have been also studied in a thin torus limit sheding some light on the origin of the appearance of liquid phases in 2D case.

At the low density limit and long range two-body interaction, liquid phases compete with the Wigner crystals (WC) [28-32]. Recently, we have shown that WC is a true ground state on a nearly flat band of Chern insulator in the limit of long range interaction and at sufficiently low densities [33]. In this work, we investigate the ground state properties of a system with interacting electrons on partially filled topological flat bands on honeycomb lattice in the thin torus limit with long range interaction. We use the exact diagonalization method to obtain the many-body ground state and analyze the crystallization strength using the pair correlation density (PCD) and its Fourier transform. We compare results for even and odd filling factors, including a scaling to a thermodynamic limit with a particle number.

\footnotetext{
* corresponding author; e-mail: michal.kupczynski@pwr.edu.pl
}

\section{Model and methodology}

We consider the Haldane model of the Chern insulator on honeycomb lattice [1]. This model is described by the following Hamiltonian:

$$
H=t \sum_{\langle i, j\rangle} a_{i}^{\dagger} a_{j}+t_{2} \sum_{\langle\langle i, j\rangle\rangle} \mathrm{e}^{\mathrm{i} \phi_{i, j}} a_{i}^{\dagger} a_{j},
$$

where $a_{i}\left(a_{i}^{+}\right)$are annhilation (creation) operators on lattice sites, $t$ and $t_{2}$ are nearest and next-nearest neighbor hoppings, respectively, and a complex phase factor $\mathrm{e}^{\mathrm{i} \phi_{i, j}}$ has the following property: $\mathrm{e}^{\mathrm{i} \phi_{i, j}}=\mathrm{e}^{-\mathrm{i} \phi_{j, i}}$ and is responsible for nontrivial topology of energy bands. To obtain nontrivial flat bands we have chosen the following values of parameters: $t=-1, t_{2}=0.31, \phi=0.65$.

We populate electrons on the lowest band on the plaquettes with $N_{x}$ and $N_{y}$ unit cells in the lattice vectors directions, respectively, and periodic boundary conditions. We fix one of the size of each plaquettes to two unit cells, $N_{x}=2$, because this is the nearest situation to the thin torus limit. We consider the screened Coulomb interaction between electrons

$$
V=\sum_{i, j} \frac{\exp \left(p-p r_{i, j}\right)}{r_{i, j}} n_{i} n_{j},
$$

where $r_{i, j}$ is the distance between sites $i$ and $j, n_{i}\left(n_{j}\right)$ is the electron density on the site $i(j)$, and $p$ is the strength of screening, which we choose to be $p=0.3$. The low energy spectrum of considered system is obtained using exact diagonalization method for different filling factors, $\nu=\frac{N}{N_{x} N_{y}}$, where $N$ is a number of particles.

To characterize the crystallization of the ground state $|\psi\rangle$ we use PCD

$$
G(i, j)=\frac{\left\langle\psi\left|a_{i}^{\dagger} a_{j}^{\dagger} a_{j} a_{i}\right| \psi\right\rangle}{\left\langle\psi\left|a_{j}^{\dagger} a_{j}\right| \psi\right\rangle} .
$$

PCD is proportional to the probability of finding a particle at a site $j$ under a condition that there is a fixed particle at a site $i$. We make PCD continuously by replacing every site by the Gaussian

$$
G_{i}(\boldsymbol{r})=\sum_{j}^{N} G(i, j) \frac{1}{2 \pi \sigma} \exp \left(\frac{\boldsymbol{r}-\boldsymbol{r}_{\boldsymbol{j}}}{2 \sigma}\right),
$$


where $\boldsymbol{r}$ is the vector connecting atom $i$ and a given point in space and $\sigma$ is the width of the Gaussian which we chose to be $\sigma=0.5$. We measure strength of the crystallization by taking the Fourier transform. We define the strength of the crystallization $S$ as a value of the highest Fourier peak excluding the peak for the zero spatial frequencies divided by the number of particles. The details of the methodology are described in Ref. [33].

\section{Results}

We calculate the ground state for many longitudinal plaquettes with filling factors from $\nu=1 / 4$ to $\nu=1 / 11$. By looking at PCD we have found that the ground state is strongly crystallized for all fillings. We can observe two patterns of that crystallization, the first one in a form of stripes (localization only in a longitudinal direction) and the second in a form of localized particles (localization in both directions), with the example shown in Fig. 1. This effect is connected with the parity of the denominator of the filling factor: stripes are present for an even denominator, and full localization for odd denominators of the filling factor. This even-odd effect is observed independently of the number of particles in the system.

(a)

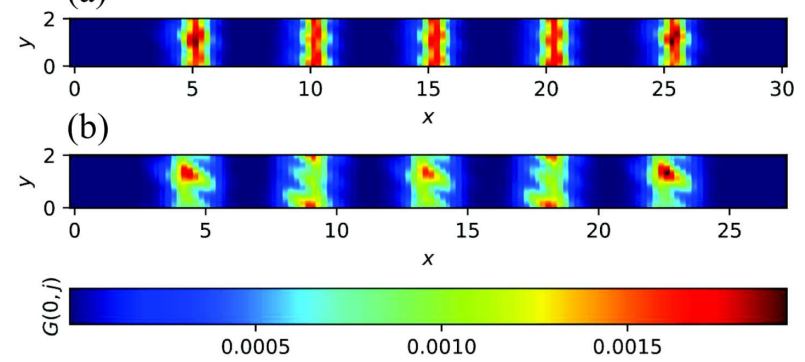

(c)

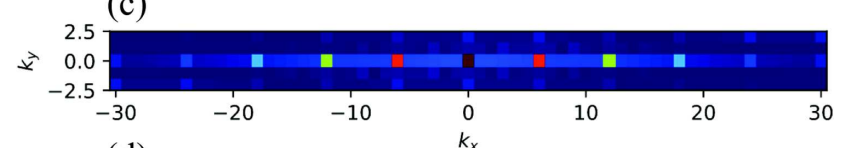

(d)

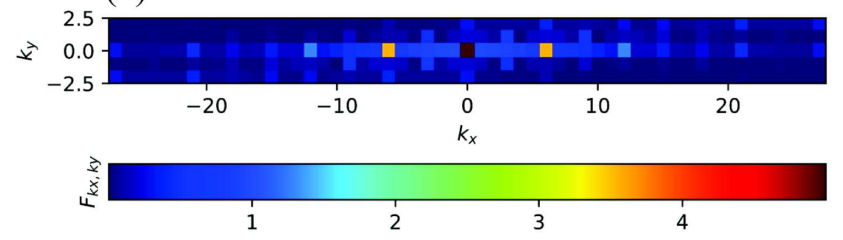

Fig. 1. The PCD of the ground state for the system with $N=6$ particles for plaquettes $N_{x} \times N_{y}=30 \times 2(\nu=$ $1 / 10)(\mathrm{a})$ and $N_{x} \times N_{y}=27 \times 2(\nu=1 / 9)(\mathrm{b})$ and their Fourier transforms in (c) and (d), respectively.

The stability of the crystallization with increasing number of particles is shown in Fig. 2. For system sizes with $1 / N<0.3$ there is no visible change in crystallization strength. One can also notice that it increases with decrease of the particle density, considering separately series with even and odd denominators of the filling factor, full and empty symbols respectively in Fig. 2.

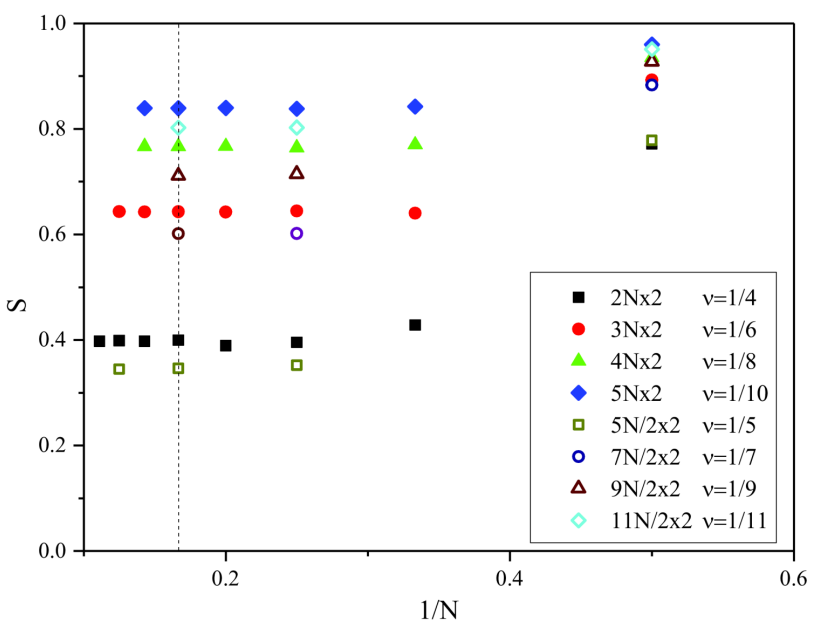

Fig. 2. The strength of the crystallization $S$ as a function of $1 / N$, where $N$ is the number of particles. Each plaquette has the following size: $N_{x} \times 2$, where $N_{x}$ depends on the filling factor $\nu$ and the number of particles $N$. The strength of crystallization is stable with increase of number of particles. The dashed line indicates plaquettes with $N=6$ particles.

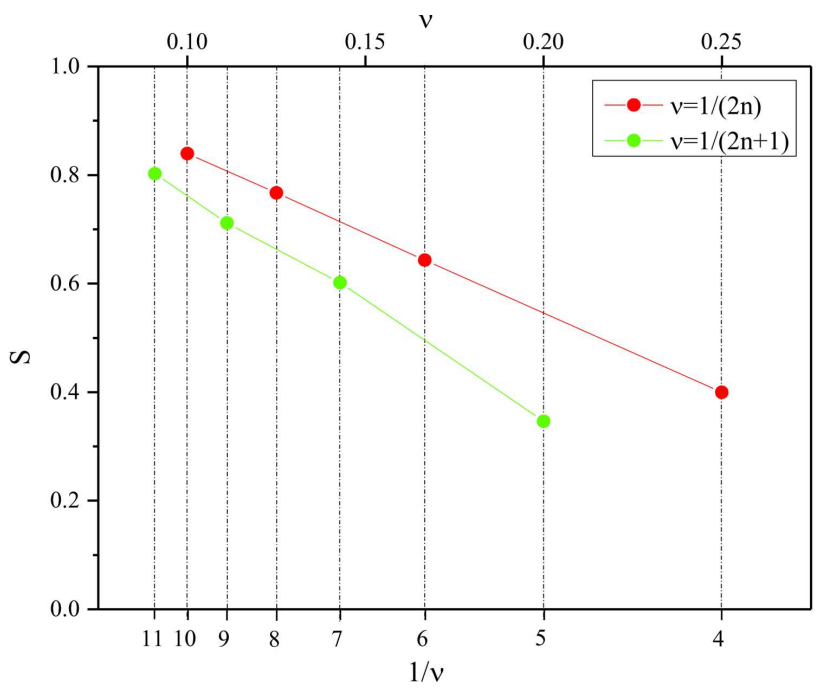

Fig. 3. The strength of the crystallization $S$ as a function of filling factor $\nu$ ( $n$ in a legend is some integer) for plaquettes with $N=6$ particles, indicated by a dashed line in Fig. 2. Two data series for the filling factors with even denominator (red line) and with odd denominator (green line) show nearly linear dependence.

To emphasize an even-odd effect, the strength of the crystallization as a function of the filling factor for these two series for a particle number $N=6$ is shown in Fig. 3 . The crystallization increases monotonically, nearly linearly, for each series when filling factor decreases. In general, the crystallization of stripes (even denominator) is stronger, in comparison to fully localized particles (odd dominator). The difference between two series disappears with lowering the filling factor. 


\section{Conclusions}

We have shown that the many-body ground state of the system on topological flat bands with screened Coulomb interaction in a thin torus limit leads to crystallization for filling factors below $\nu<1 / 4$. Two types of the crystallization are observed, for even and odd denominators of a filling factor. A partially filled band with an even denominator filling factor forms stripes, while full localization occurs for odd denominators. The crystallization is stable for different system sizes thus expected in a thermodynamic limit. The crystallization strength increases with decrease of a filling factor, and is stronger for stripes (even denominator), but the difference with fully localized particles becomes less significant for lower filling factors.

\section{Acknowledgments}

The authors acknowledge partial financial support from National Science Center (NCN), Poland, grant Maestro No. 2014/14/A/ST3/00654. Our calculations were performed at the Wrocław Center for Networking and Supercomputing.

\section{References}

[1] F.D.M. Haldane, Phys. Rev. Lett. 61, 2015 (1988).

[2] D.J. Thouless, M. Kohmoto, M.P. Nightingale, M. den Nijs, Phys. Rev. Lett. 49, 405 (1982).

[3] N.R. Cooper, N.K. Wilkin, Phys. Rev. B 60, R16279 (1999).

[4] D. Jaksch, P. Zoller, New J. Phys. 5, 56 (2003).

[5] M. Aidelsburger, M. Atala, S. Nascimbène, S. Trotzky, Y.-A. Chen, I. Bloch, Phys. Rev. Lett. 107, 255301 (2011).

[6] H. Miyake, G.A. Siviloglou, C.J. Kennedy, W.C. Burton, W. Ketterle, Phys. Rev. Lett. 111, 185302 (2013).

[7] M. Aidelsburger, M. Atala, M. Lohse, J.T. Barreiro, B. Paredes, I. Bloch, Phys. Rev. Lett. 111, 185301 (2013).

[8] G. Jotzu, M. Messer, R. Desbuquois, M. Lebrat, T. Uehlinger, D. Greif, T. Esslinger, Nature 515, 237 (2014).

[9] E. Tang, Jia-Wei Mei, Xiao-Gang Wen, Phys. Rev. Lett. 106, 236802 (2011).
[10] Kai Sun, Zhengcheng Gu, Hosho Katsura, S. Das Sarma, Phys. Rev. Lett. 106, 236803 (2011).

[11] T. Neupert, L. Santos, C. Chamon, C. Mudry, Phys. Rev. Lett. 106, 236804 (2011).

[12] D.N. Sheng, Zheng-Cheng Gu, Gu, Kai Sun, L. Sheng, Nature Commun. 2, 389 (2011).

[13] Yang-Le Wu, B.A. Bernevig, N. Regnault, Phys. Rev. B 85, 075116 (2012).

[14] N. Regnault, B.A. Bernevig, Phys. Rev. X 1, 021014 (2011).

[15] A.M. Läuchli, Zhao Liu, E.J. Bergholtz, R. Moessner, Phys. Rev. Lett. 111, 126802 (2013).

[16] S.A. Parameswaran, R. Roy, S.L. Sondhi, Compt. Rend. Phys. 14, 816 (2013).

[17] E.J. Bergholtz, Zhao Liu, Int. J. Mod. Phys. B 27, 1330017 (2013).

[18] T. Neupert, C. Chamon, T. Iadecola, L.H. Santos, C. Mudry, arXiv:1410.5828 (2014).

[19] B. Jaworowski, A. Manolescu, P. Potasz, Phys. Rev. B 92, 245119 (2015).

[20] T. Scaffidi, G. Möller, Phys. Rev. Lett. 109, 246805 (2012).

[21] Ying-Hai Wu, J.K. Jain, Kai Sun, Phys. Rev. B 86, 165129 (2012).

[22] R. Tao, D.J. Thouless, Phys. Rev. B 28, 1142 (1983).

[23] E.J. Bergholtz, J. Kailasvuori, E. Wikberg, T.H. Hansson, A. Karlhede, Phys. Rev. B $\mathbf{7 4}$, 081308 (2006).

[24] B.A. Bernevig, N. Regnault, arXiv:1204.5682, (2012).

[25] Huaiming Guo, Shun-Qing Shen, Shiping Feng, Phys. Rev. B 86, 085124 (2012).

[26] J.C. Budich, E. Ardonne, Phys. Rev. B 88, 035139 (2013).

[27] B. Jaworowski, P. Kaczmarkiewicz, P. Potasz, A. Wójs, Phys. Lett. A 382, 1419 (2018).

[28] E. Wigner, Phys. Rev. 46, 1002 (1934).

[29] N. Shibata, D. Yoshioka, J. Phys. Soc. Jpn. 72, 664 (2003).

[30] F.D.M. Haldane, Phys. Rev. Lett. 51, 605 (1983).

[31] S.A. Trugman, S. Kivelson, Phys. Rev. B 31, 5280 (1985).

[32] N. Thiebaut, N. Regnault, M.O. Goerbig, Phys. Rev. B 92, 245401 (2015).

[33] B. Jaworowski, A.D. Güçlü, P. Kaczmarkiewicz, M. Kupczyński, P. Potasz, A. Wójs, New J. Phys. 20, 063023 (2018). 